3. Serous ${ }^{5}$ or purulent otitis media can trigger polysensory hallucinations.

4. Diseased ears are oversensitive to $\mathrm{drugs}^{8,13}$ and alcohol amplifies existing otopathic hallucinations.

5. Auditory hallucinations in alcoholics arise from peripheral tinnitus.

6. Visual and auditory alcoholic hallucinations have common correlates.

7. Hallucinations and some psychotic symptoms ${ }^{7}$ arise from irritation of the peripheral audiovestibular system.

The last conclusion is the most bizarre of all, but has the considerable merit of removing the need for six other feeble, ad hoc, or desperate counter-explanations.

AG Gordon

32 Love Walk,

London SE5 8AD, England.

References

1. Carroll A, Milnes D. Unilateral auditory hallucinations in association with ear infection. Ir J Psych Med 1998; 15:31-2.

2. Sloan D, O’Boyle J. Visual alcoholic hallucinosis. Ir J Psych Med 1998; 15: 35-6.

3. Bechterew. On hallucinatory insanity following affections of the ear. J Ment Sc $1904 ; 50: 169-71$.

4. Gordon AG. Unilateral auditory hallucinations. Br J Psychiatry 1988; 153 263-4.

5. Gordon AG. Ear disease and schizophrenia - brain not needed? Acta Psychiatr Scand 1996: 93: 409-10.

6. Gordon AG. Unilateral auditory hallucinations: ear or brain? 1997; 63: 814. 6. Gordon AG. Unilateral auditory hallucinations: ear or bran? $1997 ; 63: 814$
7. Gordon AG. Insight into auditory hallucinations and psychosis. Int J Ger Psychiatry 1997; 12:410-1.

8. Gordon AG. Benzodiazepines and the ear - tinnitus, hallucinations and schizophrenia. Can J Psychiatry 1993; 38: 156-7.

9. Jaspers K. General psychopathology. Manchester: Manchester University Press, 1963.

10. McBride A, Hamilton-Kirkwood L. Unilateral auditory hallucinations. $\mathrm{Br}$ J Psychiatry 1988; 153: 264.

11. Marneros A, Beyenburg St, Berghaus A. Unilateral hallucinations and other psychotic symptoms due to otosclerosis. Psychopathology 1997; 30: 89-92.

12. Schilder P. The vestibular apparatus in neurosis and psychosis. ] Nerv Ment Dis $1933 ; 78: 1-23,137-64$

13. Gross MM, Halpert E, Sabot L. Toward a revised classification of the acute alcoholic psychoses. J Nerv Ment Dis 1967; 145: 500-8.

14. Saraway SM, Pardes H. Auditory elementary hallucinations in alcohol withdrawal psychosis. Arch Gen Psychiatry 1967; 16:653-8.

\section{Alcoholic hallucinosis revisited}

Sir - We thank Dr Gordon for his interesting comments on our case report. Firstly he quotes two previous references from the literature (Schilder 1933,' Gross 1967²) on the isolated occurrence of visual hallucination in alcoholic psychotic states, secondly he suggests that the visual hallucinations in our patient may be due to ear disease specifically vestibular dysfunction. Our literature search did not reveal the $1933^{\prime}$ and $1967^{2}$ references and we are grateful to Dr Gordon and his clearly in-depth knowledge of this topic for providing us with these references. We described the presentation of our case as "unusual in that the hallucinations described were in the visual modality only". The fact that these two previous reports date back some 65 and 31 years respectively may therefore support our contention that this presentation is unusual if not extremely rare. Fortunately we were able to obtain copies of both references. We would however have concerns firstly regarding the description by Schilder et al of 'an alcoholic with emotional deterioration' as we are not given any further information about whether he had continued to drink alcohol, whether he had ceased alcohol intake, whether he was in withdrawal or indeed would fulfill criteria for 'alcoholic hallucinosis'. Similarly we would express our concern regarding the cohort of patients described by Gross et al, specifically their finding that "hallucination in general correlated 0.48 clouding of the sensorium". Using ICD $-10^{3}$ clear consciousness is required to diagnose alcoholic hallucinosis which comes under the rubric "alcohol induced psychotic states predominantly hallucinatory" and it is not clear therefore that the cases considered by Gross satisfy modern criteria for alcoholic hallucinosis as such the cases cited above may not therefore be comparable to our individual case.

Regarding the second point Dr Gordon provides an interesting alternative explanation for the genesis of visual hallucinations in alcoholic hallucinosis citing additional case reports and references. In the case of our patient, there was no clinical evidence of vestibular dysfunction. The transient early morning retching could have represented such a symptom but had been present through much of the patients long 'drinking-life' and was much more likely to be a symptom of alcohol withdrawal. We cited a number of recent references detailing neurotransmitter dysfunction specifically hyperdopaminergic states in alcohol withdrawal which would support the development of visual hallucinations in our case. Finally, may we restate the fact that controversy surrounding the nature of alcoholic hallucinosis has been present in the literature since its first description in $1847 .{ }^{4}$

Darina Sloan, MB, MRCPSych, Senior Registrar, University College Hospital, Galway, Ireland. James O'Boyle, MB, FRCPsych, Consultant Psychiatrist,

St. Vincent's Hospital, Fairview, Dublin 3, Ireland.

\section{References}

1. Schilder P. The vestibular apparatus in neurosis and psychosis. J Nerv Ment Dis $1933 ; 78: 1-23,137-64$.

2. Gross MM, Halpert E, Sabot L. Toward a revised classification of the acute alcoholic psychosis. Arch Gen Psychiatry 1967; 16:652-8.

3. World Health Organisation. The $1 C D-10$ classification of mental and behavioural disorders, clinical descriptions and diagnostic guidelines. Geneva: WHO, 1992.

4. Marcel CNS de la folie causer par l'abus des besissiens alconliques. Thesis (Paris Rignoux, imprimeur de las faculte de medecin, 1847).

\section{Re: Unilateral auditory hallucinations}

Sir- I would like to thank Dr Gordon for bringing to our attention several cases similar to that which we reported. Over the three years which have passed since the patient in question was first admitted to hospital, it has become clear that the diagnosis is paranoid schizophrenia. The most parsimonious explanation for the symptomatology described is simply that the ear infection which coincided with the first psychotic episode exerted a pathoplastic effect on the symptomatology. Dr Gordon does not appear to have considered this possibility in the list of "bizarre or unlikely facts" which concludes his letter.

Andrew Carroll MRCPsych, Research Fellow Department of Psychiatry, The University of Edinburgh, Royal Edinburgh Hospital, Morningside Park, Edinburgh EH10 5HF, Scotland. 